\title{
Energy-Efficient Information and Communication System for Smart Meter
}

\author{
Nitin Kumar Saran
}

\begin{abstract}
The objective of this work is to develop an electricity Smart Meter, allowing the bi-directional energy flow in the Low Voltage (LV) grid. The developed prototype can be deployed in a Photovoltaic (PV) micro-production installation, being able to cooperate with the Distribution System Operator (DSO) regarding remote readings and dynamic voltage control procedures, while keeping the prosumer informed about the active power, as well as odd or faulty situations (e.g., no production). To accomplish the result proposed with functionalities that are useful in the distributed energy grid, this paper offers a device that is standard compliant by using DLMS, providing specific technical information to the DSO. Additionally, it has two low power wireless communication technologies: Bluetooth Low Energy $(B L E)$, for short range local readings and configuration by the prosumer or maintenance teams; and Sigfox, a long range low power technology, which has a permanent storage and facilitates consultation of past and current records. The latter can be accessed by an Android application from a remote location.
\end{abstract}

Keywords: Smart Meter, Smart Grid, Low Voltage Grid, PV Controller, Prosumer.

\section{INTRODUCTION}

In the last few decades, technological innovations have been sprouting at a remarkable pace, namely in the area of computer networks and information systems. This resulted in a world where humans have found innovative ways of interacting with their surroundings by building tools to help them in everyday tasks. Some of these tools are now part of what is called Internet of Things (IoT), which is comprised of smart objects that perform the most varied tasks and are able to communicate with each other and to the Internet. From this fast evolution, the outcome is the growing number of objects being connected to the Internet which brings concerns in several topics, for instance, the interoperability between different devices and manufacturers, the development of communication protocols that are adaptable to the function the devices are fabricated to perform, and the amount of energy needed to power all these new objects.

In 2010, Portugal approved ENE 20201, with the objective of being one of the countries leading the energetic revolution, through goals set to be accomplished by 2020 [1]. Some of the proposed objectives involve reaching the goal of $60 \%$ of the total produced energy being renewable, decreasing $\mathrm{CO} 2$ emissions and the overall final energetic consumption by $20 \%$, improving the economic situation of the country and the life conditions of the population. This plan includes an experiment presently being done in Évora, Portugal, named InovCity Évora, as part of the InovGrid project [2], for the development of a Smart City, with decentralized energy production and efficient consumption management using Smart Meters (SMs). This pilot is currently underway, bringing answers for the future implementation of more smart cities in the country.

The strategy above was motivated by the European Strategic Energy Technology Plan (SET Plan) which created goals for 2020, 2030 and 2050, one of them being the renovation of the present European energy infrastructure, moving towards the realization of the Smart Grid. The Smart Grid is an upgraded traditional electrical power grid, that incorporates information and communication technologies [3]. Figure 1 shows the progression of energy consumption between 1995 and 2013 in the India, revealing the growth of the consumption of renewables and gases, and the reduction of petroleum and solid fuels, confirming the work done by India.

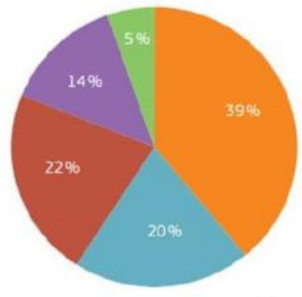

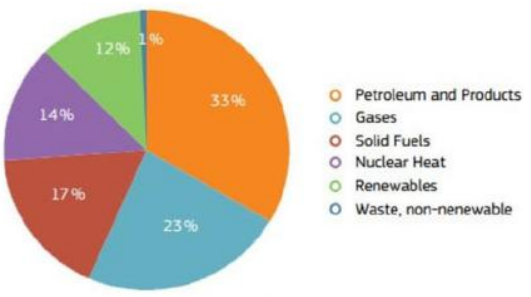

Figure 1: Gross Inland Consumption. (a) Total Primary 1995, (b) Total Primary 2013

Considering the technologies available for energy management, part of the responsibility of creating a sustainable world is being transferred back to the population by creating awareness of the problem at hand, and ultimately giving them a way of controlling the resources they are spending in their households and work environment. This is being accomplished by the increasing use of renewable energy and the monitoring and consequent adjustment of the consumption of said energy. An example of this is the installation of PV panels in common households [22].

SMs are becoming common smart devices present in residential buildings and industrial facilities. 
They have the important task of monitoring the consumption and micro-production of electricity, water and gas for a better control of the resources spent and produced, and for the detection of anomalies, equipment failure and emergency situations, issuing an alert regarding the problem at hand, so it can be promptly solved [23].

There are some papers that focus on creating solutions based on challenges energy providers possess, and establishing a commercial representation with both the consumer's and provider's interest in mind [4]. Some examples are Monitor BT and e-balance. The former, using distributed sensors, offers mechanisms for advanced fault detection and voltage control in LV grids [5]. The latter focus on integrating the consumers with the Smart Grid, using prediction models for accurate consumption behavior, allowing the creation of a business model that benefits all interested parties [6].

One of the requisites is that this SM needs to be energy efficient so that it has the least possible impact on the energy consumption. To achieve this, the communication technologies used for data transfer will have as requirement the energy efficiency. That is the case of BLE and Sigfox, the former directed to short range communication and the latter to long range communication.

A protocol that was extensively studied for the implementation in this device is the protocol defined by Device Language Message Specification (DLMS) and Companion Specification for Energy Metering (COSEM) due to being an emerging technology that is already an international standard, and is considered a powerful energy communication tool by energy distribution operators. The fact that it is an object-oriented communication protocol created exclusively for the transfer of data involving energy related information only makes it more noticeable.

\section{RELATED WORK}

\section{A. Smart Grid}

The electrical grid that presently exists has worked for a long time, but is not up to date with the modern technologies. We have been living with a grid that is composed of a one direction flow of electricity, where the population buys all the necessary resources for their daily activities, with little understanding of how taxing that is for our environment. Considering that now consumers can start producing energy in their households via renewable sources, powering said grid from the homes is now possible, promising the decrease of the use of fossil fuels and the reduction of electricity bills. That is one of the promises of the Smart Grid, the creation of a bi-directional power and data flow that is advantageous to the society [7].

As shown in Figure 2, the HAN can incorporate gateways as a mechanism that is valuable for both consumers and utilities [8]. It addresses an important topic associated to the smart home, the security. Having so many devices with communication capabilities holding personal information, makes owners vulnerable to external attacks from the internet. A gateway is a way of limiting the interaction these devices can have, since the appliances only communicate with the SM, and the SM uses the utility gateway to send the collected information to the utility servers. Furthermore, a gateway provides more options regarding the communication protocols used, since the support of IPv6 protocol is not required [9].

Most of IoT concepts can be applied to the Smart Grid, forming a more specific concept that is the Internet of Energy (IoE). The primary domains being employed in the energy sector are the control and monitoring of electrical energy using SMs, distributed energy production, and the upgrade of buildings so they are as efficient as possible, with measures as adapting light intensity, or turning it off completely, according to preset parameters such as occupancy and location.

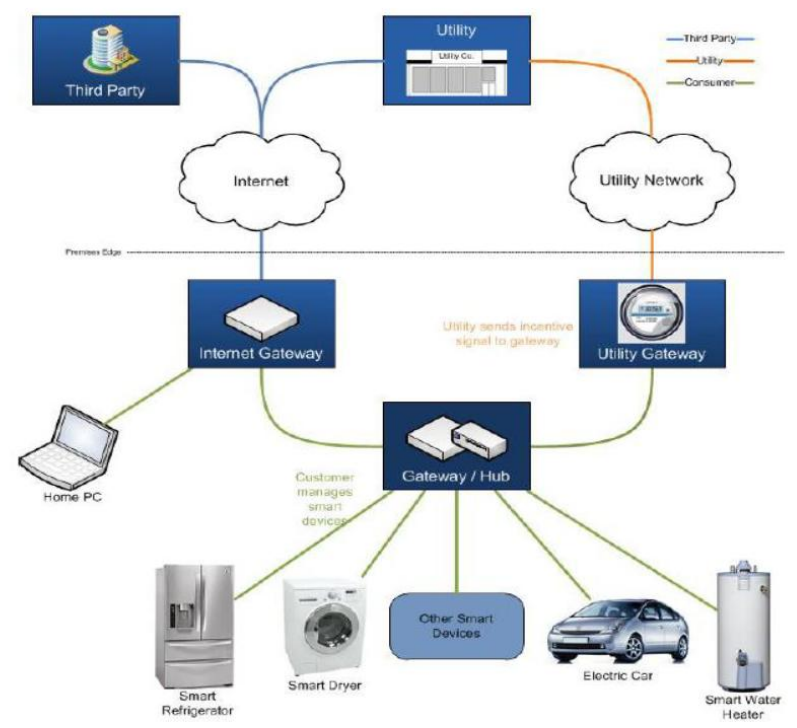

Figure 2: Consumer's Smart Devices [10].

\section{B. : IoT Technologies}

Wireless technologies connect heterogeneous objects for a complete integration of the services provided, offering the resources needed to platforms, so that data consumers can interact remotely with smart appliances. These protocols offer mobility and flexibility to those who use them, and are responsible for providing remote access to homeowners, utilities and third party service providers for a better incorporation of SMs in the Smart Grid. This chapter introduces some IoT communication protocols, both short range and long range, providing a description of their features and main functionalities [23].

\section{Smart Metering Communication}

This section introduces a brief analysis of an emerging smart metering communication protocol, DLMS/COSEM. In this paper DLMS/COSEM is used as the protocol for energy information exchange, using bursts of data to accomplish it.

Communication technologies in the context of AMIs tend to follow a few guidelines for their implementation to be considered. 
The measured data, whether it be energy, power, voltage or volume needs to be supported and there is a requirement for integrity of the transferred data, clock synchronization for the dynamic switch of tariff based on timetables, firmware update to fix eventual vulnerabilities or to add/improve features [11].

The energy market requires a big amount of information to be retrieved from energy meters so that the billing process and spending estimations can be as accurate as possible. This stresses the importance of a protocol that not only can collect data in different formats but is adaptable to future necessities, such as the addition of more functionalities.

DLMS and COSEM define this protocol that is based on object modelling techniques, including object and interface classes. IEC 62056 is a series of standards for electricity metering data exchange and is structured based on a client-server model in which requests are made by the client and the corresponding data are replied by the SM [12]. These standards are the international version of DLMS/COSEM.

\section{Commercial Smart Meters}

The first energy metering devices had as their only feature, the measurement of energy spent during periodic intervals. The readings were executed manually by competent personnel, and was an inefficient and time consuming task. With the evolution of these devices some features were added to improve the reading procedure. At first were introduced meters equipped with a radio transmitter, then with PLC and mobile data, becoming the meters we have in our homes today [13]. However, they do not have the necessary characteristics for their integration in the Smart Grid.

The installation of SMs in a big scale is a result of the pursuit of energy efficiency, bringing benefits to consumers, utilities and the environment. On the consumer side, the users will be able to have detailed information about their energy usage, leading to the adjustment of their habits, and bills will be based on actual consumption, not estimations. For utilities, remote readings allow more accurate billing and enable dynamic tariffs, power outages and peak demands are reduced, resulting in an efficient use of power resources [14]. Itron's Smart Grid Solution is shown in figure 3.

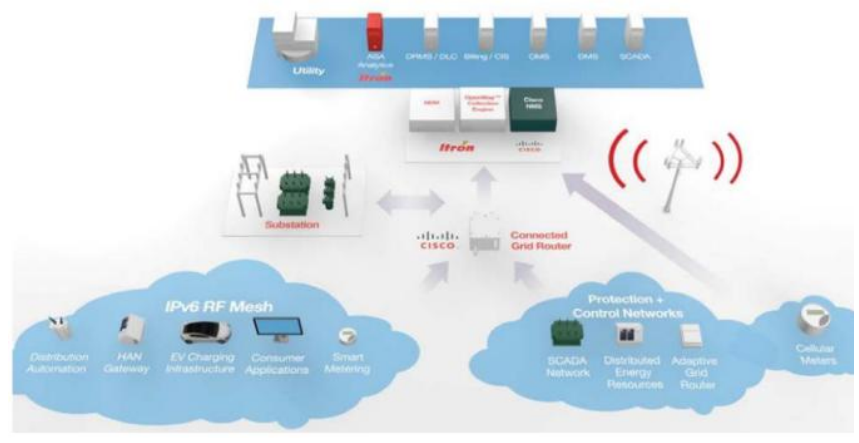

Figure 3: Itron's Smart Grid Solution [15].

\section{Problem Solution}

\section{A. Monitoring of Voltage and Current in the LV Grid}

This paper is inserted in the context of Smart Grid applied research, aims to solve a significant and specific problem, that is remote LV grid monitoring and control. This has gained significantly more importance due to the need to insert distributed energy micro-generation into the grid. But these technological improvements only came to emphasize all the problems that were already present such as lack of means to monitor the LV grid and ability to detect LV faults.

Unlike the High Voltage (HV) and the Medium Voltage (MV) grids, the traditional LV grid is seriously undeveloped (it should be noted that the LV grid is the most braided part of the energy grid) and as such, does not have any mechanisms to actively regulate voltage and adapt to topology changes, which if implemented would give more control to the DSO as well as being more reliable to the end user.

An important service that needs to be supported is the voltage control of the active power injected by PV systems, which is now possible through the automation of distribution and monitoring that is now part of the responsibility of secondary substations [16]. The importance of this service is due to the voltage instability caused by the bi-directional energy flows in power grids with Distributed Energy Resource (DER) penetration, which are gradually becoming widespread.

For the accomplishment of such solution, new equipment is needed for functions such as information gathering and exchange in order to be able to report the operational status of the grid, as well as actuation mechanisms to convey set points to remote PV systems. This paper proposes a SM that is able not only to perform local readings on behalf of a user, but also support communication between the Distribution Transformer Controller (DTC) and the local PV controller or inverter, for an automated regulation of the PV micro-generation and peak demands [17]. The DTC is installed in every Secondary Substation and acts as local metering, monitoring and automation devices.

The SM will be closely coupled with the PV unit set, since it will be located near a PV node. Figure 4 shows the overall operating environment in which the developed SM is to be integrated.

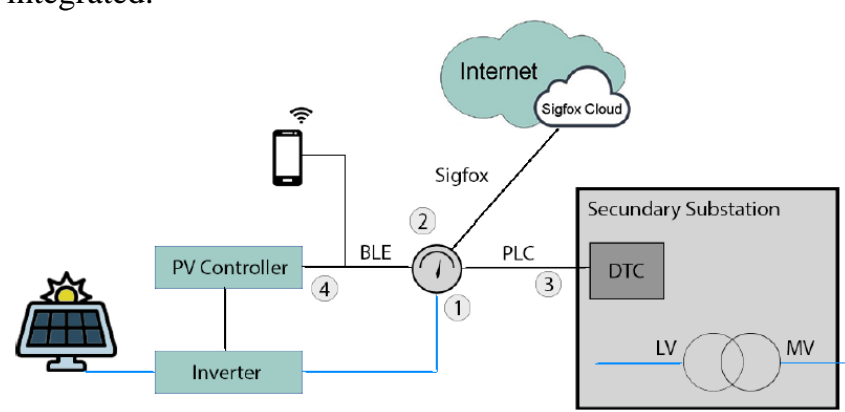

Figure 4: LV monitoring and control system with SM.

The following steps demand the direct involvement of the SM. They portray a sequence beginning when the sensor in the SM conveys the voltage, current and active power readings to the closed-loop voltage control system.

1. The SM performs regular energy readings, approximately once every 5 seconds;

2. These values can be read locally by the prosumer, maintenance teams and the PV controller via BLE, or remotely by the prosumer, using Sigfox;

3. Via DLMS over PLC, the DTC will regularly enquire the SM of the

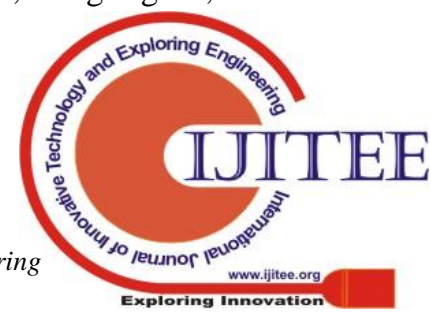


values that were measured. It will also check the $\mathrm{LV}$ grid power flow in order to calculate the power set point for each PV node, sending this information back to the SM.

4. Via BLE, the PV controller can read the adjustments to be made and perform the necessary actions by automatically limiting the active power being injected by the PV inverter, as per the DTC instructions.

Solutions like the one being here proposed need to be studied and implemented, due to the trend of massification of PV micro-production [24].

The solution proposed in this paper recommends the standards and technologies to be used so that an effective communication can be performed with different key devices in the LV grid, offering the possibility of adaptation to more specific requirements or circumstances without losing focus on one important factor, which is unit cost.

\section{B. Smart Meter}

The developed SM prototype aggregates several technologies in order to create a functional and innovative device, being suitable to both industrial and home installations. The data that are currently measured are the following: voltage, current and real power. These can be read using any of the following communication technologies: DLMS over serial port (e.g., PLC modem), BLE and Sigfox. Figure 19 is a photograph of the developed SM prototype, with all the associated components.

The board used as platform to the development of the proposed SM was the Akeru 3.3, which is an Arduino board. This board comes with a Sigfox modem and a half-wave antenna, and were added a BLE module, a push button, and an energy sensor to shape the SM that was designed. Figure 5 shows the smart meter developed.

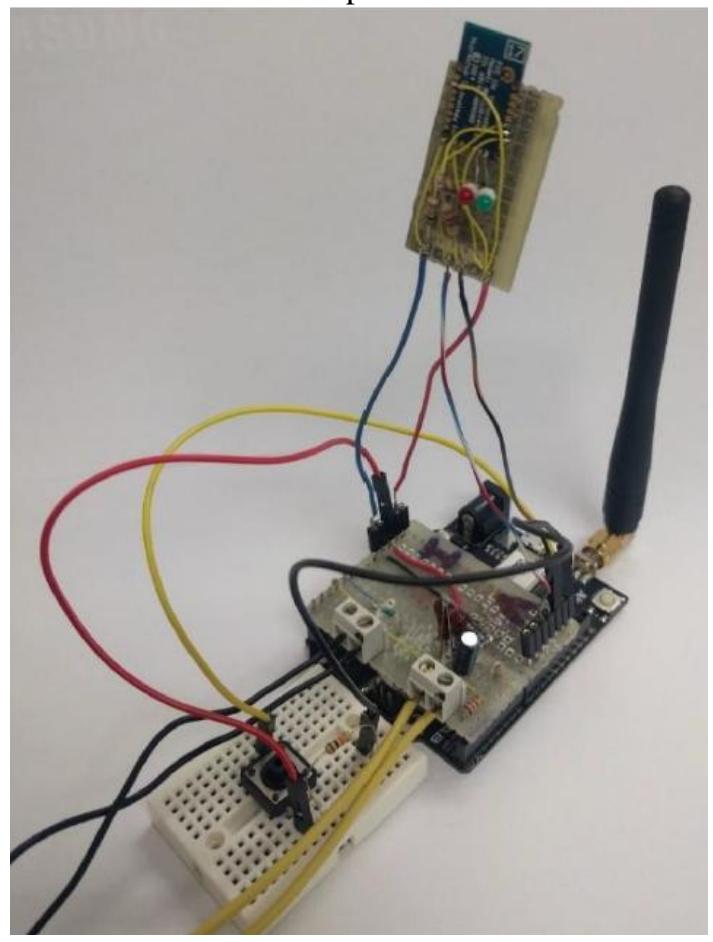

Figure 5: Smart Meter developed

Figure 6 shows the energy module that was used to measure the voltage, current and power, and was created specifically for the Arduino used in this paper. The use of this module was fundamental for the tests performed and a great tool to draw accurate graphs. For the software component, Open Energy Monitor [18] provides open source monitoring and their library for energy readings was used, offering the measurement of several variables, such as Vrms, Irms, real power, apparent power and power factor.

Figure 7 contains a flowchart of the program created, giving a visual aid in understanding the overall process involved. As can be observed, the flowchart depicts a sequence of events that runs through all the technologies used in the paper. It was developed this way because it only has one task done regularly that is the BLE data update. The Sigfox transfer only occurs in periods of 30 minutes or in case of detection of abnormal values, and the DLMS transfer in a periodicity defined by the DTC but not frequent in nature. This means that only when one of these last communications is occurring that some small delay may happen, but otherwise the expected duration of the cycle is not disturbed [21].
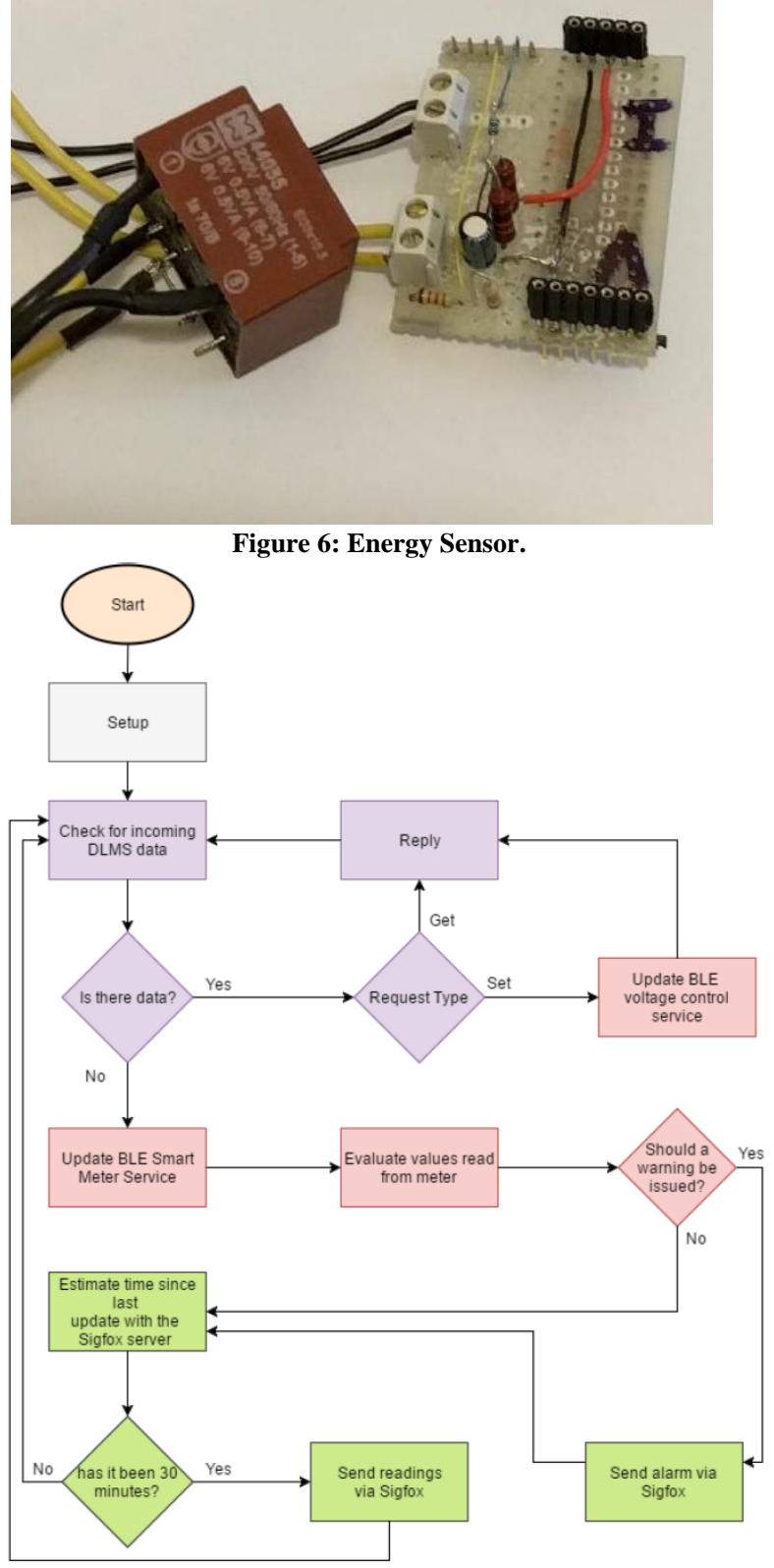

Figure 7: Flowchart of the program. 
To provide a viable communication between a client and server, with verification of the validity of the replies delivered, this specific server followed the message exchange available in the Gurux client application example. This application can send all the client messages that are needed for a simple yet successful and informational data exchange, and the answers that the server must create to correctly reply were formed with the aid of the books of the DLMS User Association.

As per the DLMS specifications, the client is the one that initiates the connection with the server, so the Arduino continually checks for incoming data via the serial port, specifically searching for the initial delimiter character of a DLMS frame.

When the device recognizes that a DLMS frame is being received, the content of the message is stored and each byte is processed. The processing phase of a frame includes, among others, the verification of its validity and the identification of the type of content available in the message, rapidly distinguishing between the various kinds of requests available All the valid frames that are possible to create with this protocol follow a strict structure and order that needs to be fulfilled in order to be possible the proper interpretation of the message.

Figure 8 shows an overview of the data exchange related to the OBIS codes requests and replies, identifying the two main components of an object: OBIS and attribute, which identify the type of object and the kind of information that is needed. The figure also refers to another subroutine that is detailed later in this section.

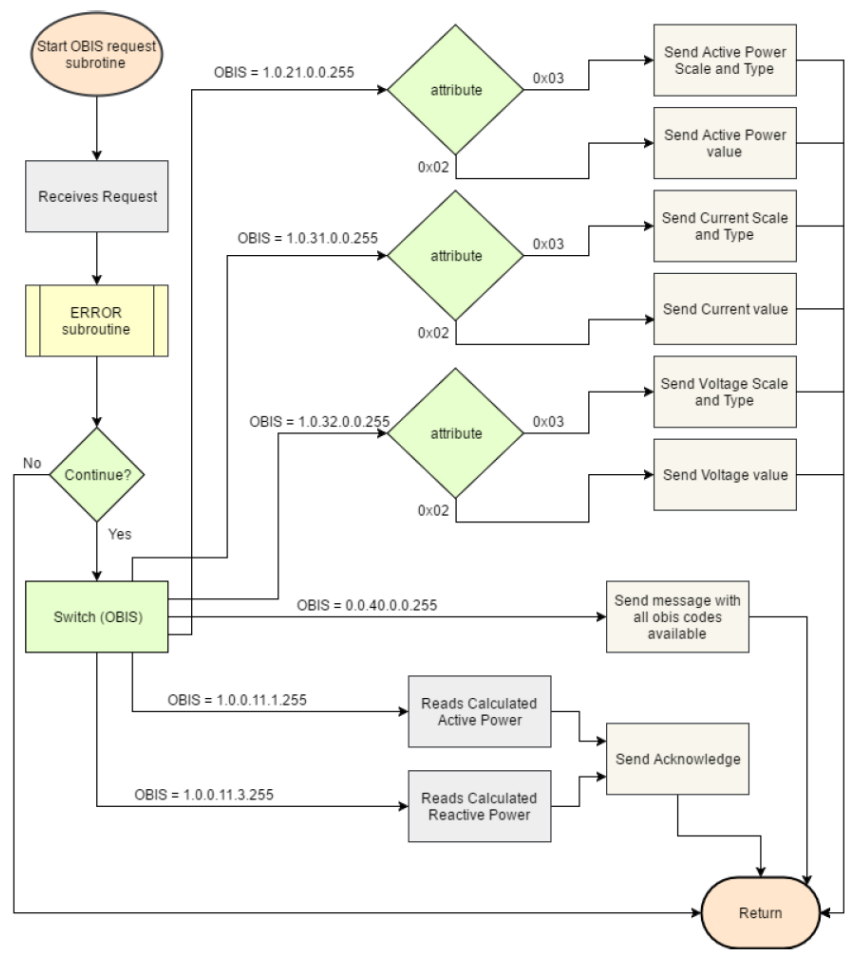

Figure 8: OBIS codes implemented.

Since not all clients are equal, nor all servers, some error messages were programmed in the server so that if it receives a message that it does not recognize, an appropriate reply is transmitted and the connection can continue (see Figure 9 for the error messages implemented). It should be noted that a default client application only waits a variable pre-programmed amount of time without receiving a valid message before dropping a connection.

In this server, data blocks are not available since at the time of completion of the program the client example application did not support it. Although it is now supported by the example used, it is not a demanded feature by any DLMS client so an error message was implemented to inform the client that a single block request needs to be sent. The other error messages inform the client that the requested object or attribute (i.e., value or scale) is not available.

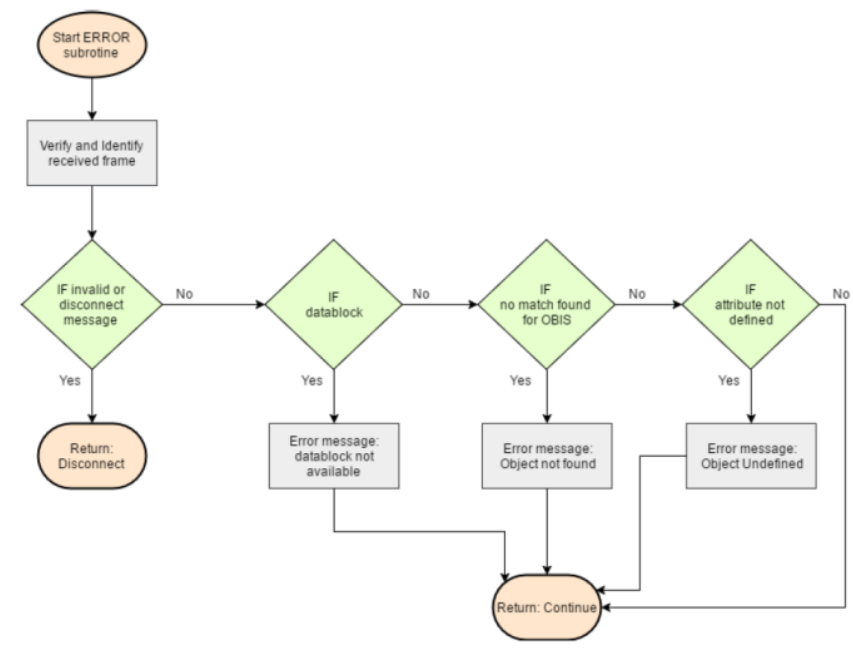

Figure 9: DLMS - Error messages implemented.

\section{Evaluation of Proposed Solution}

\section{A. Hardware}

The hardware used in this project was chosen considering the functionality it was required and availability for testing purposes at the time. The general characteristics that were taken into account in the choice of hardware were energy efficiency, cost and ease of use. For each technology, a set of more specific attributes were considered.

Developer board: The Arduino board was not the only one considered for this project, but was the one that met all the criteria established. For a better understanding of the line of thought, a brief summary of those other technologies is included. Figure 10 shows two developer boards, one an Arduino and the other a Raspberry Pi.
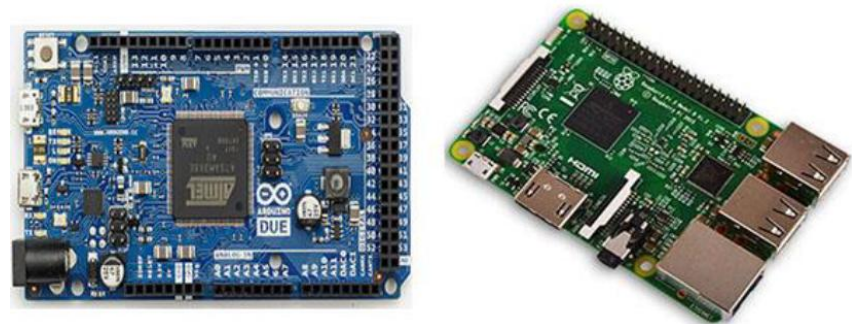

Figure 10: (a) Arduino Due; (b) Raspberry Pi 3 Model B.

While Raspberry Pi is a single-board computer, both Arduino and STM32 are micro-controllers. Each of these devices should be used according to the type of project one is trying to develop. In the case of this dissertation, we need a device that can easily read sensors, can execute communications using different protocols, is power efficient and low cost. 
Using Raspberry Pi to perform these tasks would be more complex for the analog reading necessary and would require more interaction once operational, and it has a higher power consumption and cost. All these requirements point to the use of a micro- controller instead of any Raspberry Pi in the market, limiting the final choice to an Arduino or a STM32 board.

Between Arduino and a STM32 board, both are highly capable of offering the attributes necessary to the development of this project so the decision comes greatly from the factor that, at the time of the testing of this technology, the acquisition of a Sigfox module for single and personal use was more complicated and expensive than large scale buying, and the same for the yearly subscription. It was available the Akeru 3.3 board, which is an Arduino board, with a Sigfox module and antenna already integrated that facilitated the accomplishment of the proposed SM prototype. It is important to note that this project could be done in either of these families of boards, if the minimum requirements to run the program are fulfilled. Table 1 shows the minimum requirements.

Table 1: Characteristics of Previous Monitoring Systems [19]

\begin{tabular}{|l|l|l|}
\hline Type & Min. required & Used \\
\hline Flash Memory & $32 \mathrm{~KB}$ & $17.618 \mathrm{~B}$ \\
\hline RAM & $2 \mathrm{~KB}$ & $1.488 \mathrm{~B}$ \\
\hline Digital I/O Pins & 6 & 6 \\
\hline Analog Input Pins & 2 & 2 \\
\hline
\end{tabular}

An assessment of the price of these components and the overall cost of the developed SM was done, in order to obtain a cost appraisal.

Sigfox module: A LPWAN communication technology is a key point in this project, since it provides reliable data independently of where it is located and without the need to build a supporting network. Three technologies that fit the criteria were studied, but Sigfox was the one that provided the solution that was more adaptable to the plan.

At this point in time, NB-IoT is not commercially available but is an emerging powerful technology that should be carefully considered for future projects that involve Smart Grid or IoT devices. LoRaWAN also is still a recent technology that does not have the wide coverage provided by Sigfox.

Sigfox was considered the most advantageous LPWAN solution for this implementation. With several deciding factors distinguishing both, the two that were more significant were the slightly higher cost of LoRaWAN and the fact that there is no LoRaWAN operator in Portugal at the time of conclusion of this project.

For the Sigfox appraisal, several shops were found and each offered different prices and set of components. Although there are several different modules available, since they offer the same functionalities, only the least expensive component of each needed type was included, and they were separated into categories. For a complete assessment, the cost of a yearly subscription was needed but these values are not available online for the common user. It is expected that large scale buying reduces the unit cost very significantly.

In the development of this project an Akeru 3.3 was used,

since it already has Sigfox incorporated and a valid subscription offered by Narrownet, the Sigfox operator in Portugal. The Figure 11 shows an image of the board used.

BLE module: A low power, short range communication technology was considered for the purpose of facilitating the transfer of small amounts of data for quick consultations or adjustments. The technologies considered were ZigBee and BLE, and an assessment of both was conducted. BLE was indeed the most suitable choice considering it is already integrated in smartphones.

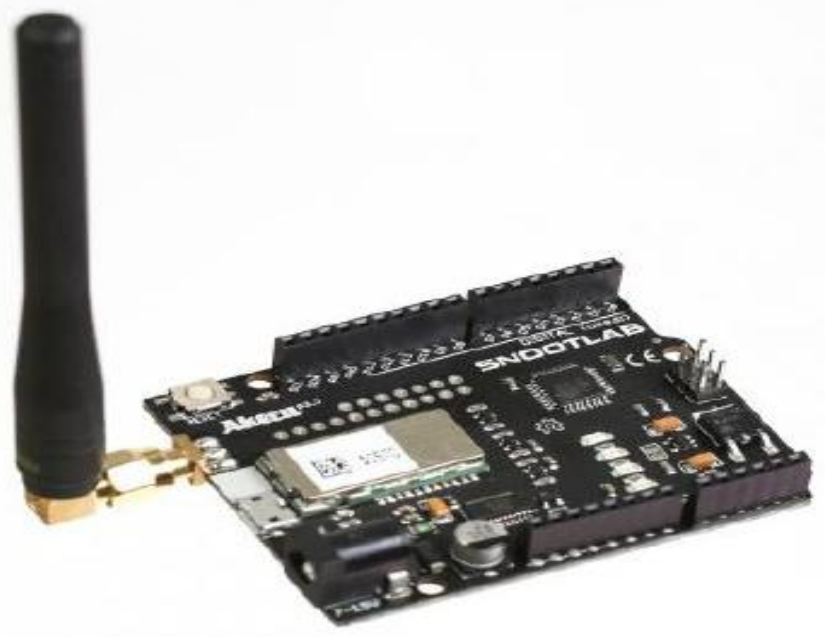

Figure 11: Akeru 3.3 [20].

There are currently many manufacturers for BLE modules, and choosing a specific model was not an easy task. One of the features that were a determining factor was the support of an asynchronous serial data connection between devices, for the possibility of implementing remote configurations on the BLE module or even serial communications, if the situation requires it. This meant that the pool of possible modules was shortened since Microchip is the only manufacturer that provides MLDP, which is a proprietary protocol for this type of communication.

Considering only Microchip modules that support MLDP, the two most adequate and cost efficient are the RN4020 and the RN4871 (see Figure 12), both having similar characteristics and compatibility with each other. The latter is presented as the next-generation BLE device with lower power consumption and improvement in the data throughput.
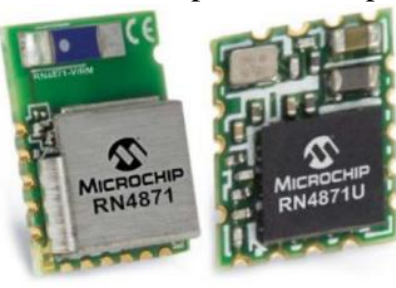

(a)

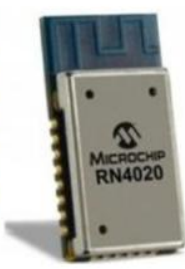

Figure 12: Microchip (a) RN4871; (b) RN4020.

Final solution: The overall cost of the final solution is calculated having into account the least expensive components that are or could be integrated without significant changes in the performance or consumption of this project. It is later compared with existing SMs so that the value present can be related with existing solutions.

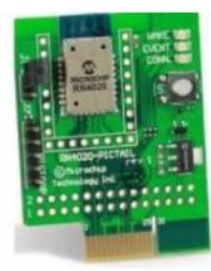

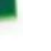


Although these components are not the ones used in the solution of this project, they offer the same features and are in the same price range so that the solution is expected to perform the same as the ideal solution projected above. The reason for this change is the fact that some of the used components were the ones currently available in the market or were the best way to test a specific technology, given the circumstances.

To provide a way of determining if the cost of the proposed meter is affordable, a market analysis was conducted to evaluate the price range of the products available in online shops. These devices, like the prototype developed, are single phase, but lack any wireless communication technology (except infrared), preferring Modbus or RS485, and many do not support DLMS.

\section{V.CONClusions AND Future SCOPE}

Considering the current opportunities for the installation of energy micro-generation systems in houses and commercial buildings, innovative ways of monitoring and controlling the bi-directional energy flow in the LV grid need to be created, thus allowing for an effective organization of the prosumers production and consumption by the DSO aiming for the grid stability. This is the case of fault detection in the LV grid and topology changes when they are needed.

This was accomplished by implementing several innovative communication technologies to transfer data in the most efficient and adequate way for each required exchange. Therefore, DLMS, a standard for energy communication was implemented for transfer of data with the DSO via a serial interface (ex.: PLC). BLE, a low power short range technology was employed to make available regular instantaneous energy readings to be consulted by the prosumer, and instructions sent by the DSO to be read by the PV controller. And Sigfox, a low power long range communication technology, that stores average voltage and current readings for an analysis to the balance of energy consumption and generation, and generates alarms of abnormal situations.

The work done in this paper should be continued, although the performance of the Sigfox technology was appropriate to this solution, it is important to perform comparative testing with other LPWANs, for an evaluation of these innovative communication technologies, that at this moment do not have the necessary coverage to effectively apply in a project. Furthermore, the exploration of the development of algorithms for voltage control of grids with PV systems is important and constitute a central part of the SM deployment in Smart Grids.

\section{REFERENCES}

1. Resolução do Conselho de Ministros no 29/2010, "Plano Energértico 2020,” Diário da Répública n.o 73 , Ia série 15 Abril 2010, vol. 2020, no. Ene 2020, pp. 1289-1296, 2010.

2. EDP Distribuição, "InovGrid." [Online]. Available: http://www.edpdistribuicao.pt/pt/rede/InovGrid/Pages/InovGrid.asp x. [Accessed: 03-Aug-2019].

3. A. Hafeez, N. H. Kandil, B. Al-Omar, T. Landolsi, and A. R. Al-Ali, "Smart home area networks protocols within the smart grid context," J. Commun., vol. 9, no. 9, pp. 665-671, 2014.
4. European Commission, "EU Energy in Figures - Statistica Pocketbook 2015,” 2015. [Online]. Available: http://ec.europa.eu/transport/facts-fundings/statistics/doc/2015/pb 2015- section26.xlsx. [Accessed: 03-Aug-2019].

5. A. Bernardo et al., "Monitor BT Pilot Project: Combined Voltage Regulation Approach for LV Grids with PV Penetration," 23rd Int. Conf. Electr. Distrib., pp. 15-18, 2015.

6. B. E. Matusiak, K. Piotrowski, and J. S. Zieliński, "Internet of Things in e-balance project," Zarządzanie energią i teleinformatyka (ZET 2015), pp. 98-103, 2015.

7. N. Binti and M. Isa, "Smart Grid Technology: Communications , Power Electronics and Control System," 2015 Int. Conf. Sustain. Energy Eng. Appl., pp. 10-14, 2015.

8. S. Clements, T. Carroll, and M. Hadley, "Home Area Networks and the Smart Grid," U.S. Dep. Energy, 2011.

9. T. Mendes, R. Godina, E. Rodrigues, J. Matias, and J. Catalão, "Smart Home Communication Technologies and Applications: Wireless Protocol Assessment for Home Area Network Resources," Energies, vol. 8, no. 7, pp. 7279-7311, 2015.

10. M. Erol-Kantarci and H. T. Mouftah, "Energy-Efficient Information and Communication Infrastructures in the Smart Grid: A Survey on Interactions and Open Issues," IEEE Commun. Surv. Tutorials, vol. 17, no. 1, pp. 179-197, 2015.

11. S. Feuerhahn, M. Zillgith, C. Wittwer, and C. Wietfeld, "Comparison of the Communication Protocols DLMS / COSEM , SML and IEC 61850 for Smart Metering Applications," 2011 IEEE Int. Conf. Smart Grid Commun., pp. 410-415, 2011.

12. G. Kmethy, "IEC 62056 DLMS/COSEM - How to accommodate new requirements while maintaining interoperability," 2009. [Online]. Available:

http://www.dlms.com/downloads/dlms_bangkok_gk090511.pdf. [Accessed: 03-Aug-2019].

13. N. S. Živi, O. Ur-rehman, C. Ruland, S. Member, A. M. Meter, and R. Mmr, "Evolution of Smart Metering Systems," Telecommun. forum, no. 23rd, pp. 635-638, 2015.

14. G. R. Barai, S. Member, S. Krishnan, S. Member, B. Venkatesh, and S. Member, "Smart Metering and Functionalities of Smart Meters in Smart Grid," pp. 138-145.

15. Prysmian, "Smart Grid Solutions," Smart Grid Solut. Guid., p. 19, 2014.

16. A. Al-Fuqaha, M. Guizani, M. Mohammadi, M. Aledhari, and M. Ayyash, "Internet of Things: A Survey on Enabling Technologies, Protocols and Applications," IEEE Commun. Surv. Tutorials, vol. 17, no. 99, pp. 2347-2376, 2015.

17. M. S. Nunes et al., "Leveraging Fault Detection and Voltage Control in Low Voltage Grids Based on Distributed Monitoring," CIRED Work. 2014, no. 337, pp. 1-5, 2014.

18. "Home | OpenEnergyMonitor." [Online]. Available: https://openenergymonitor.org/. [Accessed: 25-July-2019].

19. C. Beard and W. Stallings, Wireless Communication Networks and Systems, 1st ed. Pearson Education, 2015.

20. Snootlab, “Akeru beta 3.3." [Online]. Available: http://snootlab.com/lang-en/snootlabshields/829-akeru-beta-33-en.html. [Accessed: 30-July-2019].

21. E. U. Week et al., "Huawei and Janz CE Announce the First Smart Electrical Energy Meter Based on NB-IOT," 2017.

22. GSM Association, "3GPP Low Power Wide Area Technologies (White Paper)," 2016.

23. LoRa Alliance, "A technical overview of LoRa and LoRaWAN," no. November, pp. 1-20, 2015.

24. A. J. Jara, D. Fernandez, P. Lopez, M. A. Zamora, A. F. Skarmeta, and L. Marin, "Evaluation of bluetooth low energy capabilities for tele-mobile monitoring in home-care," J. Univers. Comput. Sci., vol. 19, no. 9, pp. 1219-1241, 2013. 